ITP-UH-25/98

November 1998

\title{
A GENERALIZED SPIN LADDER IN A MAGNETIC FIELD
}

\author{
Holger Frahm用 and Claus RöDEnBECK \\ Institut für Theoretische Physik, Universität Hannover \\ D-30167 Hannover, Germany
}

\begin{abstract}
We study the phase diagram of coupled spin-1/2 chains with bilinear and (chiral) three-spin exchange interactions in a magnetic field. The model is soluble on a one-parametric line in the space of coupling constants connecting the limiting cases of a single and two decoupled Heisenberg chains with nearest neighbour exchange only. We give a complete classification of the low-energy properties of the integrable system and introduce a numerical method which allows to study the possible phases of spin ladder systems away from the soluble line in a magnetic field.
\end{abstract}

PACS-Nos.: 75.10.Jm 75.30.Kz 75.50.Ee

*e-mail: frahm@itp.uni-hannover.de 


\section{Introduction}

One dimensional quantum spin systems have attracted much interest in recent years. On the theoretical side, soluble models and powerful methods such as bosonization combined with numerical results have been used to understand many aspects of the rich physics found in these systems. In particular the Bethe Ansatz solution of the $S=1 / 2$ Heisenberg chain [1] with nearest neighbour interaction has provided much insight into their properties. At the same time the fabrication of materials which are essentially quasi-one dimensional spin-1/2 systems allows for experimental studies of these features. In addition to nearest neighbour exchange the effects of competing next-nearest neighbour interactions driving a spin Peierls transition [2 [4] and interchain interaction as in spin ladders [5 8] have been considered to account for the properties of these compounds. Furthermore multi-spin exchange terms can be included without breaking the $S U(2)$ symmetry of the system [9 11]. In general such terms are known to drive phase transitions in the Heisenberg chain opening a gap $\Delta$ between the singlet ground state and the lowest (triplet) excitation.

New interesting properties can be observed when such systems are placed in a magnetic field [12,13]: For $H>\Delta / g \mu_{B}$ the system undergoes a transition from the gapped phase to an incommensurate phase with non-zero magnetization in which it has gapless excitations until the field is strong enough to lead to a saturated ferromagnetic state. Signatures of these transitions and properties of the gapless high-field phase have already been studied in several organic $S=1 / 2$ spin Peierls and ladder systems 114 [16.

The subject of this paper is to study of the properties of this gapless phase. Our starting point is a soluble model of coupled spin-1/2 Heisenberg chains $(0 \leq \kappa \leq 1)$ 17 20

$$
\begin{aligned}
& \mathcal{H}= \sum_{i=1}^{N}\left\{J_{1}\left(\vec{S}_{2 i-1} \vec{S}_{2 i}+\vec{S}_{2 i} \vec{S}_{2 i+1}\right)+J_{2}\left(\vec{S}_{2 i} \vec{S}_{2 i+2}+\vec{S}_{2 i+1} \vec{S}_{2 i+3}\right)\right. \\
&\left.+J_{3}\left(\vec{S}_{2 i-1}\left(\vec{S}_{2 i} \times \vec{S}_{2 i+1}\right)-\vec{S}_{2 i}\left(\vec{S}_{2 i+1} \times \vec{S}_{2 i+2}\right)\right)\right\} \\
& J_{1}=2 J(1-\kappa), \quad J_{2}=J \kappa, \quad J_{3}=2 J \sqrt{\kappa(1-\kappa)} .
\end{aligned}
$$

The three-spin exchange term guarantees integrability of the system for arbitrary ratio of antiferromagnetic pair exchange constants, i.e. $0<\kappa<1$ (for more general cases see e.g. 21]). It breaks parity and time-reversal invariance, for a possible mechanism for the appearence of such a term see Ref. [22]. Below we put $J=1$ which fixes our scale of energy. In the following section we use the Bethe Ansatz solution to give a detailed account of the magnetic phase diagram and the critical properties of this model. Unlike the systems discussed above the model (1.1) has gapless excitations for any $\kappa$ as long as the magnetization is not saturated. 
Furthermore, for sufficiently large $\kappa$ we find a transition into a second critical phase with different universality class at an intermediate magnetic field [20]. Based on these findings we introduce a numerical method for the determination of the phase boundaries which allows to extend this discussion to nonintegrable generalizations of (1.1). Finally we apply this method to propose a phase diagram of the system with competing nearest and next-nearest neighbour exchange (i.e. without the three spin terms in (1.1)).

\section{The integrable model}

Starting from the ferromagnetic eigenstate with all spins up the spectrum of the integrable Hamiltonian (1.1) is obtained by adding magnons which are parametrized by the roots of the Bethe-Ansatz equations (BAE) [17]

$$
\left(\frac{\lambda_{j}-\tilde{\kappa}+\frac{i}{2}}{\lambda_{j}-\tilde{\kappa}-\frac{i}{2}}\right)^{N}\left(\frac{\lambda_{j}+\tilde{\kappa}+\frac{i}{2}}{\lambda_{j}+\tilde{\kappa}-\frac{i}{2}}\right)^{N}=\prod_{j \neq k}^{M} \frac{\lambda_{j}-\lambda_{k}+i}{\lambda_{j}-\lambda_{k}-i}
$$

with $2 \widetilde{\kappa}=\sqrt{\kappa /(1-\kappa)}$. The corresponding state has magnetization $\mathcal{M}=N-M$ and energy

$$
E\left(\left\{\lambda_{j}\right\}\right)=\pi \sum_{j=1}^{M}\left(a_{1}\left(\lambda_{j}+\tilde{\kappa}\right)+a_{1}\left(\lambda_{j}-\tilde{\kappa}\right)\right), \quad \pi a_{n}(\lambda)=-\frac{2 n}{4 \lambda^{2}+n^{2}} .
$$

Generic solutions to the BAE are arranged in $m$-strings of complex rapidities $\lambda_{j}^{(m)}=x+i((m+$ $1) / 2-j), j=1, \ldots, m$ with common real part. For the ground state only real solutions to (2.1) have to be taken into account. In the thermodynamic limit the ground state in a magnetic field $H=h / g \mu_{B}$ is obtained by filling all states with negative dressed energy, defined in terms of the integral equation

$$
\epsilon_{1}(\lambda)+\int_{\epsilon(\lambda)<0} d \mu a_{2}(\lambda-\mu) \epsilon(\mu)=h+\pi\left(a_{1}(\lambda+\tilde{\kappa})+a_{1}(\lambda-\tilde{\kappa})\right) .
$$

For $h=0$ one finds $\epsilon_{1}<0$ for all $\lambda$, the corresponding ground state is parameterized uniquely by $N$ real rapidities, giving a singlet state with energy $(\Psi(x)$ is the digamma function)

$$
\frac{E}{N}=-\ln 2-\frac{1}{4}\left\{\Psi(1-i \tilde{\kappa})-\Psi\left(\frac{1}{2}-i \tilde{\kappa}\right)+\Psi(1+i \tilde{\kappa})-\Psi\left(\frac{1}{2}+i \tilde{\kappa}\right)\right\} .
$$

The low-lying excitations are scattering states of spinons [23] and the low energy behaviour of the theory is that of a level-1 $S U(2)$ Wess-Zumino-Witten model with central charge $c=1$ independent of $\kappa<1$ [24]. A small magnetic field breaks the $S U(2)$ symmetry giving a $c=1$ 
Gaussian conformal field theory (CFT) with anomalous dimensions depending on $h$. Increasing the magnetic field $h$ beyond

$$
h \geq h_{c 2}= \begin{cases}4(1-\kappa) & \text { for } 0 \leq \kappa \leq \frac{1}{4} \\ 1+\kappa^{-\frac{1}{2}} & \text { for } \frac{1}{4} \leq \kappa \leq 1\end{cases}
$$

one has $\epsilon_{1}(\lambda)>0$ resulting in a ferromagnetically polarized ground state 20.

For $\kappa>1 / 4$ an intermediate phase can be found for sufficiently strong fields $h_{c 1}<h<h_{c 2}$ which is characterized by

$$
\epsilon_{1}(\lambda)<0 \text { for } \Lambda_{1}<|\lambda|<\Lambda_{2}
$$

$\left(\Lambda_{1,2}\right.$ have to be determined as functions of the magnetic field from (2.3).) Here the ground state is formed by filling two 'Fermi seas' of magnons with positive (negative) rapidities $\lambda$. The presence of gapless excitations near the Fermi points at $\lambda= \pm \Lambda_{1}, \pm \Lambda_{2}$ changes the low energy spectrum of the system which in this region has to be described in terms of two $c=1$ Gaussian CFTs [20] very similar to a Luttinger liquid of electrons in one spatial dimension [25, 26]. The asymptotic behaviour of correlation functions can be determined from the finite-size corrections to the energy of low lying states. Following Refs. [27, 28] we obtain (see Appendix A)

$$
\Delta E=-\frac{\pi}{6 N^{2}}\left(v_{1}+v_{2}\right)+\frac{2 \pi}{N^{2}}\left\{v_{1}\left(\Delta_{1}^{+}+\Delta_{1}^{-}\right)+v_{2}\left(\Delta_{2}^{+}+\Delta_{2}^{-}\right)\right\}
$$

where $v_{1,2}$ are the Fermi velocities (A.6) of excitations near the points $\Lambda_{1,2}$ and

$$
\begin{aligned}
& \Delta_{1}^{ \pm}=\frac{1}{2}\left\{\frac{\left(x_{12} \Delta M_{2}-x_{22} \Delta M_{1}\right)}{2(\operatorname{det} \mathbf{X})} \mp \frac{\left(z_{12} \Delta D_{2}-z_{22} \Delta D_{1}\right)}{2(\operatorname{det} \mathbf{Z})}\right\}^{2}, \\
& \Delta_{2}^{ \pm}=\frac{1}{2}\left\{\frac{\left(x_{11} \Delta M_{2}-x_{21} \Delta M_{1}\right)}{2(\operatorname{det} \mathbf{X})} \mp \frac{\left(z_{11} \Delta D_{2}-z_{21} \Delta D_{1}\right)}{2(\operatorname{det} \mathbf{Z})}\right\}^{2}
\end{aligned}
$$

are the conformal dimensions of the primary fields in the two Gaussian models expressed in terms of $2 \times 2$-matrices $\mathbf{X}$ and $\mathbf{Z}$ with elements (A.4) and (A.5) respectively. The integers $\Delta M_{i}$ and $\Delta D_{i}$ denote the difference of the quantum numbers (A.2) between the excitation and the ground state.

Taking the limit $h \searrow h_{c 1}$ corresponding to $\Lambda_{1}=0$ the matrix elements of $\mathbf{X}$ and $\mathbf{Z}$ can be expressed in terms of the scalar dressed charge $\xi$ satisfying the integral equation $\xi(\lambda)=$ $1-\int_{-\Lambda_{2}}^{\Lambda_{2}} d \mu a_{2}(\lambda-\mu) \xi(\mu)$ as

$$
\mathbf{X}=\left(\begin{array}{cc}
1 & 0 \\
1-\xi(0) & \xi\left(\Lambda_{2}\right)
\end{array}\right), \quad \mathbf{Z}=\frac{1}{\xi\left(\Lambda_{2}\right)}\left(\begin{array}{cc}
\xi\left(\Lambda_{2}\right) & 1-\xi(0) \\
0 & 1
\end{array}\right)
$$

\footnotetext{
${ }^{1}$ In other systems allowing for several types of massless excitations the relation $\mathbf{X} \propto \mathbf{Z}^{-1}$ allows to parameterize the critical exponents in terms of a single dressed charge matrix 25, 26, 29, 30. Contrary to our claim in Ref. [20] this is not possible in this model.
} 
This allows to simplify the expressions for the conformal dimensions (2.8) for $h=h_{c 1}+0$ as

$$
\begin{aligned}
& \Delta_{1}^{ \pm}=\frac{1}{8}\left\{\Delta M_{1} \pm\left[(1-\xi(0)) \Delta D_{2}-\Delta D_{1}\right]\right\}^{2} \\
& \Delta_{2}^{ \pm}=\frac{1}{8}\left\{\frac{(1-\xi(0)) \Delta M_{1}-\Delta M_{2}}{\xi\left(\Lambda_{2}\right)} \pm \xi\left(\Lambda_{2}\right) \Delta D_{2}\right\}^{2}
\end{aligned}
$$

From (2.7) the correlation function of the operator with quantum numbers $\Delta M_{i}, \Delta D_{i}$ is found to decay algebraically as $(1 / x)^{\alpha}$ with an exponent $\alpha=2\left(\Delta_{1}^{+}+\Delta_{1}^{-}+\Delta_{2}^{+}+\Delta_{2}^{-}\right)$[25, 26]. Due to the nature of the ground state in the regime $h_{c 1}<h<h_{c 2}$ several low energy processes contributing to $C^{z z}(x)=\left\langle S^{z}(x) S^{z}(0)\right\rangle$ have to be considered (see Fig. 1). The corresponding quantum numbers in $(2.8)$ are

$$
\begin{array}{lllll}
\text { (1) } \Delta D_{1}=0 & \Delta D_{2}=2 & \Delta M_{1}=0 & \Delta M_{2}=0 \\
\text { (2) } \Delta D_{1}=-1 & \Delta D_{2}=1 & \Delta M_{1}=1 & \Delta M_{2}=1 \\
\text { (3) } \Delta D_{1}=1 & \Delta D_{2}=1 & \Delta M_{1}=1 & \Delta M_{2}=1 \\
\text { (4) } \Delta D_{1}=2 & \Delta D_{2}=0 & \Delta M_{1}=0 & \Delta M_{2}=0
\end{array}
$$

The leading asymptotic behaviour of $C^{z z}(x)$ is determined by the smallest exponent $\alpha$. Approaching the transition line $h=h_{c 1}(\kappa)$ from above we find that this exponent corresponds to the process (1) for $1 / 4<\kappa<\kappa_{c}\left(h_{c 1}\right)$ and $(3)$ for $\kappa_{c}\left(h_{c 1}\right)<\kappa<1$. At $\kappa=\kappa_{c}\left(h_{c 1}\right) \approx 0.61$ or $h_{c 1}\left(\kappa_{c}\right) \approx 1.35$ a crossover from an effective single chain to two chain behaviour takes place. Comparing the resulting exponents $\alpha$ to the one obtained in the single Gaussian theory for $h \nearrow h_{c 1}$ [31] we find from (2.10)

$$
\alpha= \begin{cases}2 \xi\left(\Lambda_{2}\right)^{2} & 0 \leq h<h_{c 1} \\ 2(1-\xi(0))^{2}+2 \xi\left(\Lambda_{2}\right)^{2} & h \searrow h_{c 1}, \kappa<\kappa_{c}\left(h_{c 1}\right) \\ \frac{1}{2}\left(1+\xi\left(\Lambda_{2}\right)^{-2}\right)\left(\xi\left(\Lambda_{2}\right)^{2}+\xi(0)^{2}\right) & h \searrow h_{c 1}, \kappa>\kappa_{c}\left(h_{c 1}\right)\end{cases}
$$

This crossover in the long distance asymptotics of $C^{z z}(x)$ in the high field phase with $h_{c 1}<h<$ $h_{c 2}$ can be observed for any $\kappa>\kappa_{c}\left(h_{c 1}\right)$ : in the limit $\kappa \rightarrow 1$ of decoupled chains the exponents due to the most relevant processes (1) and (3) in (2.11) are $\alpha^{(1)}=1 / \xi(\Lambda)^{2}$ and $\alpha^{(3)}=2 \xi(\Lambda)^{2}$ where $\Lambda=\left(\Lambda_{2}-\Lambda_{1}\right) / 2$. Again the dominant process at low energies is determined by the effective single-chain process with exponent $\alpha^{(3)}$ for small fields with a crossover to the twochain process $(1)$ at a magnetic field to be determined from $2 \xi(\Lambda)^{4}=1$, or $h \approx 1.29$. Solving the integral equations numerically we obtain the complete phase diagram of the integrable chain in a magnetic field (Fig. 2). Depending on $\kappa$ and $h$ the low energy properties of the system are those of a single or of two Gaussian CFTs. Increasing the magnetic field in the latter regime a crossover is observed corresponding a change from the intrachain process (3) to the interchain 
process (1) as the most important one at low energies. The typical dependence of the exponent $\alpha$ for fixed ratio of the exchange constants on the magnetic field is shown in Fig. 3 .

\section{Phase diagram of generalized spin ladders}

To investigate whether the magnetic phases found in the integrable system (1.1) persist when we relax the conditions on the pair and three spin exchange constants we consider the Hamiltonian (1.1) with general values of the exchange constants $J_{i}$. For magnetic fields strong enough to polarize the system completely (corresponding to $h_{c 2}$ in the previous section) the excitations above the ferromagnetic ground state are spin waves. Their dispersion is easily obtained to be

$$
E_{ \pm}=J_{2} \cos k \pm \sqrt{J_{1}^{2} \cos ^{2}(k / 2)+\left(J_{3}^{2} / 4\right) \sin ^{2} k}+\text { const. }
$$

Below $h_{c 2}$ the ferromagnetic state becomes unstable against creation of these magnons. Depending on the coupling constants $J_{i}$ one finds either a single magnon mode at $k=0$ or two modes with wave numbers $\pm Q$ becoming soft at the transition into the paramagnetic phasejust as in the integrable model discussed in the previous section. The transition between these two scenarios occurs at the point where the low energy magnon modes (3.1) have a quartic dispersion $E_{-} \sim k^{4}$ for small wave numbers. This requires a ratio

$$
\frac{J_{2}}{J_{1}}=\frac{1}{4}\left(1-\frac{J_{3}^{2}}{J_{1}^{2}}\right)
$$

for the pair exchange constants. For the integrable case (1.1) this is the point $(\kappa, h)=(1 / 4,3)$ in the phase diagram Fig. 2, i.e. the upper end point of the transition line between the two gapless phases. For a model without three spin exchange terms $\left(J_{1}, J_{2}, J_{3}\right)=(2(1-\kappa), \kappa, 0)$ Eq. (3.2) implies a similar transition for $\kappa=1 / 3$ or $J_{2} / J_{1}=1 / 4$ (see also below).

For magnetic fields $h<h_{c 2}$ computation of spin wave dispersions as in (3.1) is not sufficient due to strong quantum fluctuations in one spatial dimension. A possible criterion to determine the phase boundary at smaller fields is the field dependence of the zero temperature magnetization which shows a characteristic singularity on the transition line [20]. However, while this feature can be identified easily in the thermodynamic limit accessible for the Bethe Ansatz soluble model (1.1) it cannot be used to analyze the numerical data due to the discrete set of magnetizations realized in a finite size system. Similarly, the dramatic changes in the low energy spectrum from a single Gaussian model to the form (2.7) are difficult to see from the finite size data obtained by numerical diagonalization.

As a possible method to identify the transition line $h_{c 1}$ from numerical finite size data we propose the following method: having identified the phase for small ratios $J_{2} / J_{1}$ as similar in 
nature with the single Heisenberg chain $J_{2}=0=J_{3}$ we compute the overlap

$$
\mathcal{O}\left(J_{1}, J_{2}, J_{3}\right) \equiv\left|\left\langle\left\{J_{1}, 0,0\right\} \mid\left\{J_{1}, J_{2}, J_{3}\right\}\right\rangle\right|
$$

between the finite size ground states $\left|\left\{J_{1}, J_{2}, J_{3}\right\}\right\rangle$ of (1.1) with fixed magnetization and the corresponding one of the Heisenberg chain. Clearly, this quantity will decrease from the value $\mathcal{O}\left(J_{1}, 0,0\right)=1$ for increasing next nearest neighbour and three spin interactions. In Fig. 4 we present data for (3.3) obtained for the integrable system (1.1) with 16 spins in the sectors with different magnetization showing a sharp transition as a function of $\kappa$. As shown in Fig. 2 these transitions provide an excellent numerical estimate of the critical field $h_{c 1}$.

Away from the integrable line this sharp transition of the overlap (3.3) as a function of $J_{2} / J_{1}$ is smoothed out. However, there are still well defined inflection points of the function $\mathcal{O}\left(J_{1}, J_{2}, J_{3}\right)$ along lines in the space of parameters $J_{2,3}$. This allows to estimate the position $h_{c 1}$ of the transition in the nonintegrable model. In Fig. 5 the resulting phase diagram is shown for the system with nearest and next nearest neighbour pair exchange only, i.e. $\left(J_{1}, J_{2}, J_{3}\right)=(2(1-$ $\kappa), \kappa, 0)$. Without an external magnetic field this model is known to have gapless excitations for $0 \leq J_{2} / J_{1} \lesssim 0.241$ and in the limit of decoupled chains $J_{1} \rightarrow 0$ [ [2]. For intermediate values of the next nearest neighbour exchange the system has a spin gap $\Delta$ leading to a plateau in at $M^{z}=0$ in the magnetization curve extending to $h=\Delta$. For strong magnetic fields $h \sim h_{c 2}$ the spin wave result (3.2) shows a transition from a phase with one and two types of magnons with quadratic dispersion, respectively. For intermediate fields the $\kappa$-dependence of $\mathcal{O}(2(1-\kappa), \kappa, 0)$ allows to locate the transition between these phases which is found to end near $\kappa \approx 0.5$ at the transition into the gapped phase.

\section{Summary and Conclusion}

To summarize we have presented a detailed account of the magnetic phase diagram of an integrable model (1.1) of generalized coupled spin chains. We have established two distinct zero temperature phases which can be described in terms of a single and two Gaussian conformal field theories respectively. The exact finite-size corrections in the spectra of low energy excitations in the latter phase allow to classify the possible critical exponents arising in the long distance asymptotics of two-point correlation functions. As an example the exponent in $C^{z z}(x)$ has been found to show a strong dependence on the magnetic field. In the same correlator we have found a crossover from an intrachain to an interchain process as the most relevant one at low energies. 
Finally we have extended our discussion to the possible magnetic phases of the system (1.1) with general exchange constants, in particular the spin chain with competing nearest and next-nearest pair exchange only. Combining known results on the nature of the zero field ground state with spin wave calculations and numerical simulations using the overlap (3.3) as

a criterion for the transition we have obtained the phase diagram of this system. Assuming that the phase found at large $\kappa$ is of similar nature as in the integrable model (this is certainly true in the large field limit) this gives strong constraints on a proper bosonization of the model in the gapless phase found for $h>\Delta$. We should note, however, that by construction our analysis can not exclude the existence of additional phases at larger values of $J_{2} / J_{1}$ (which are not present in the integrable model). An study in the limit of two weakly coupled chains $\left(J_{1} \ll J_{2}\right)$ in a magnetic field may give additional insights on this region.

\section{Acknowledgments}

This work has been supported by the Deutsche Forschungsgemeinschaft under Grant No. Fr $737 / 2-3$.

\section{A Finite size spectrum of the integrable model}

To compute the finite-size spectrum of the integrable model in the regime $h_{c 1}<h<h_{c 2}$ we consider solutions of the BAE (2.1) where all possible real solutions in the intervals $\mathcal{I}_{-}=$ $\left[\Lambda_{2}^{-}, \Lambda_{1}^{-}\right]$and $\mathcal{I}_{+}=\left[\Lambda_{1}^{+}, \Lambda_{2}^{+}\right]$with $\Lambda_{1}^{-}<\Lambda_{1}^{+}$are present (see Refs. [27, 28]). Their density satisfies the equation

$$
\rho(\lambda)+\left\{\int_{+}+\int_{-}\right\} d \mu a_{2}(\lambda-\mu) \rho(\mu)=a_{1}(\lambda+\tilde{\kappa})+a_{1}(\lambda-\tilde{\kappa}) .
$$

where $\int_{ \pm}$denotes integration over the interval $\mathcal{I}_{ \pm}$. From $\rho$ we define the quantum numbers (related to total magnetization and momentum) of the corresponding state as

$$
m_{i}=\frac{M_{i}}{N}=\int_{\Lambda_{i}^{-}}^{\Lambda_{i}^{+}} d \mu \rho(\mu), \quad d_{i}=\frac{D_{i}}{N}=\left(\int_{-\infty}^{\Lambda_{i}^{-}}-\int_{\Lambda_{i}^{+}}^{\infty}\right) d \mu \rho(\mu) .
$$

To express the finite size corrections to the energy of this state in terms of these quantum numbers, the matrix $\partial\left\{m_{i}, d_{i}\right\} / \partial\left\{\Lambda_{j}^{+}, \Lambda_{j}^{-}\right\}$has to be computed. Defining function $g(\lambda \mid \Lambda)$ with

$$
g(\lambda \mid \Lambda)+\left\{\int_{+}+\int_{-}\right\} d \mu a_{2}(\lambda-\mu) g(\mu \mid \Lambda)=a_{2}(\lambda-\Lambda)
$$


and restricting ourselves to the case $\Lambda_{i}^{ \pm}= \pm \Lambda_{i}$ relevant for the Hamiltonian (1.1) we obtain

$$
x_{i j} \equiv \pm \frac{1}{\rho\left(\Lambda_{j}\right)} \frac{\partial m_{i}}{\partial \Lambda_{j}^{ \pm}}=\delta_{i j}-(-1)^{j} \int_{-\Lambda_{i}}^{\Lambda_{i}} d \mu g\left(\mu \mid \Lambda_{j}\right)
$$

and

$$
z_{i j} \equiv \frac{1}{\rho\left(\Lambda_{j}\right)} \frac{\partial d_{i}}{\partial \Lambda_{j}^{ \pm}}=\delta_{i j}-(-1)^{j}\left(\int_{-\infty}^{-\Lambda_{i}}-\int_{\Lambda_{i}}^{\infty}\right) d \mu g\left(\mu \mid \Lambda_{j}\right) .
$$

Following Woynarovich 28] these identities yield (2.7) and (2.8) with the Fermi velocities

$$
v_{i}=\left.(-1)^{i} \frac{1}{2 \pi \rho\left(\Lambda_{i}\right)} \frac{\partial \epsilon_{1}(\lambda)}{\partial \lambda}\right|_{\lambda=\Lambda_{i}} .
$$

\section{References}

[1] H. Bethe, Z. Phys. 71, 205 (1931).

[2] G. Castilla, S. Chakravarty, and V. J. Emery, Phys. Rev. Lett. 75, 1823 (1995).

[3] J. Riera and S. Koval, Phys. Rev. B 53, 770 (1996).

[4] S. R. White and I. Affleck, Phys. Rev. B 54, 9862 (1996), cond-mat/9602126.

[5] T. Barnes, E. Dagotto, J. Riera, and E. S. Swanson, Phys. Rev. B 47, 3196 (1993).

[6] S. Gopalan, T. M. Rice, and M. Sigrist, Phys. Rev. B 49, 8901 (1994).

[7] D. G. Shelton, A. A. Nersesyan, and A. M. Tsvelik, Phys. Rev. B 53, 8521 (1996), cond-mat/9508047.

[8] S. Brehmer, H.-J. Mikeska, and U. Neugebauer, J. Phys. Condens. Matter 8, 7161 (1996), cond-mat/9604136.

[9] A. A. Nersesyan and A. M. Tsvelik, Phys. Rev. Lett. 78, 3939 (1997), cond-mat/9612014, ibid. 79, 1171(E) (1997).

[10] A. K. Kolezhuk and H.-J. Mikeska, Phys. Rev. Lett. 80, 2709 (1998), cond-mat/9712087.

[11] A. K. Kolezhuk and H.-J. Mikeska, preprint (1998), cond-mat/9803176.

[12] R. Chitra and T. Giamarchi, Phys. Rev. B 55, 5816 (1997), cond-mat/9611114.

[13] M. Usami and S. Suga, preprint (1998), cond-mat/9809088.

[14] V. Kiryukhin, B. Keimer, and D. E. Moncton, Phys. Rev. Lett. 74, 1669 (1995).

[15] G. Chaboussant et al., Phys. Rev. Lett. 79, 925 (1997), cond-mat/9706138.

[16] G. Chaboussant et al., Phys. Rev. Lett. 80, 2713 (1998), cond-mat/9711124.

[17] V. Yu. Popkov and A. A. Zvyagin, Phys. Lett. A 175, 295 (1993).

[18] A. A. Zvyagin, JETP Lett. 60, 580 (1994), [Pis'ma Zh. Eksp. Teor. Fiz. 60, 563-568 (1994)]. 
[19] H. Frahm and C. Rödenbeck, Europhys. Lett. 33, 47 (1996).

[20] H. Frahm and C. Rödenbeck, J. Phys. A 30, 4467 (1997), cond-mat/9702083.

[21] S. Park and K. Lee, J. Phys. A 31, 6569 (1998).

[22] T. Okamoto and S. Kawaji, J. Phys. Soc. Japan 65, 3716 (1996).

[23] L. D. Faddeev and L. A. Takhtajan, J. Sov. Math. 24, 241 (1984), [Zap. Nauch. Semin. LOMI 109, 134 (1981)].

[24] I. Affleck, Field theory methods and quantum critical phenomena, in Fields, Strings and Critical Phenomena, edited by E. Brézin and J. Zinn-Justin, pp. 563-640, Les Houches, Session XLIX, North-Holland, 1990.

[25] H. Frahm and V. E. Korepin, Phys. Rev. B 42, 10553 (1990); ibid. 43, 5653 (1991).

[26] N. Kawakami and S.-K. Yang, J. Phys. Condens. Matter 3, 5983 (1991).

[27] H. J. de Vega and F. Woynarowich, Nucl. Phys. B 251, 439 (1985).

[28] F. Woynarovich, J. Phys. A 22, 4243 (1989).

[29] A. G. Izergin, V. E. Korepin, and N. Yu. Reshetikhin, J. Phys. A 22, 2615 (1989).

[30] H. Frahm and N.-C. Yu, J. Phys. A 23, 2115 (1990).

[31] N. M. Bogoliubov, A. G. Izergin, and V. E. Korepin, Nucl. Phys. B 275 [FS17], 687 (1986). 


\section{Figures}

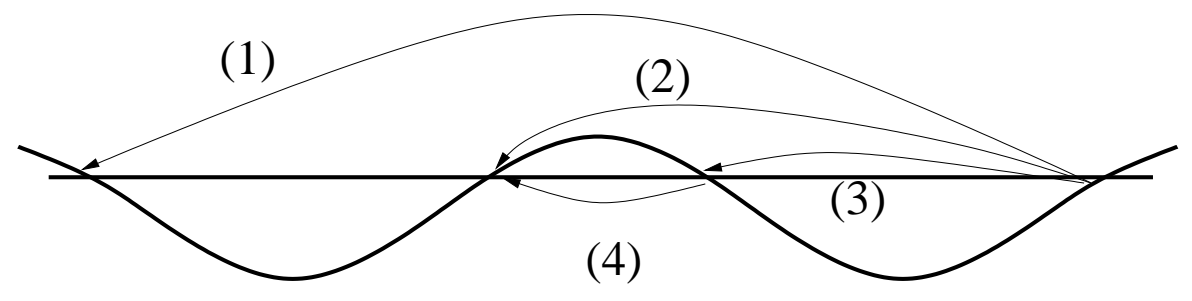

Figure 1: Low-energy processes contributing to $C^{z z}(x)$.

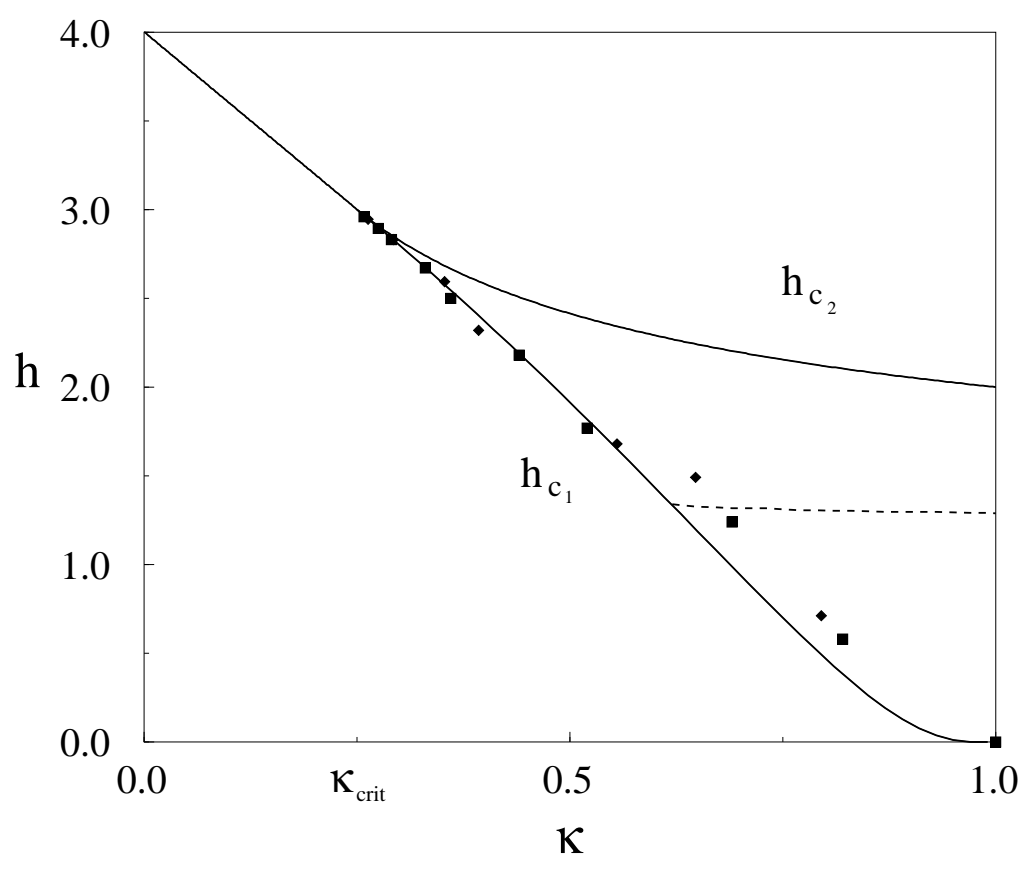

Figure 2: Magnetic phase diagram of the integrable model. The dashed line marks the crossover between the intrachain and interchain process as dominant low energy contribution to $C^{z z}$, the symbols denote the position of the phase transition as determined from numerical diagonalization of systems with 16 and 20 spins using the singularities in the operlaps (3.3). 


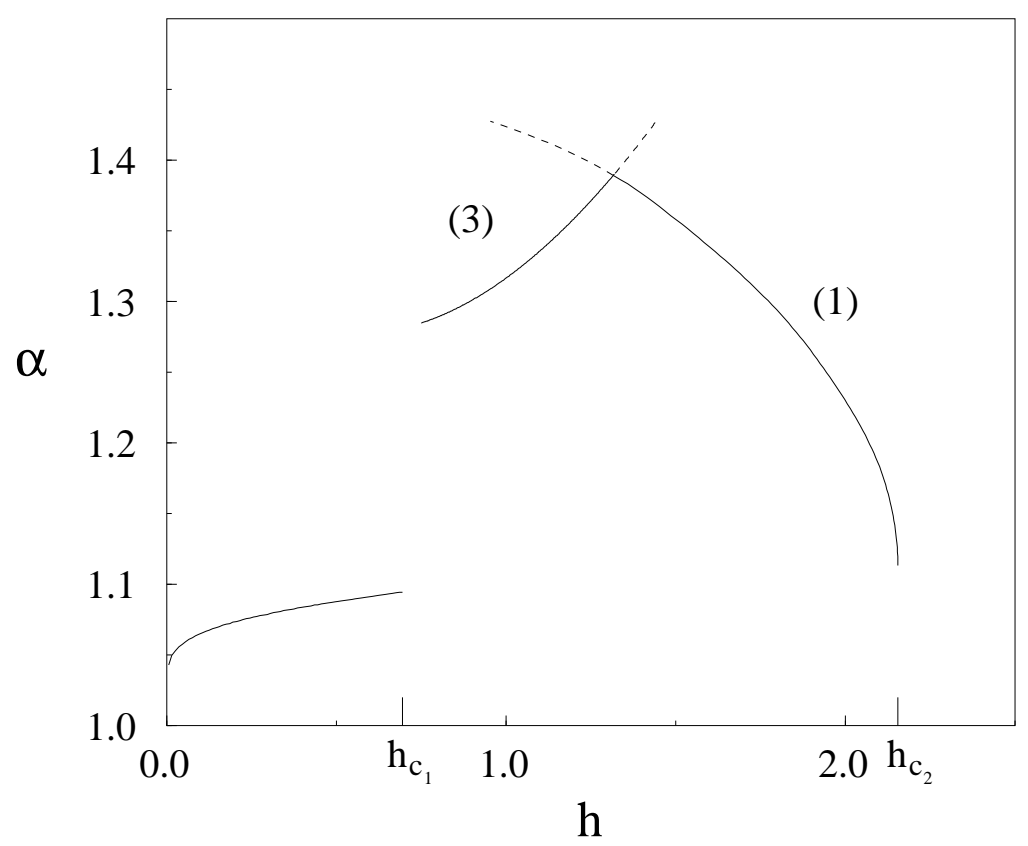

Figure 3: Critical exponent $\alpha$ of $C^{z z}(x)$ for $\kappa=3 / 4$ as a function of the magnetic field. The cusp at $h \approx 1.31$ is a consequence of the crossover from the intrachain (3) to the interchain process (1) in Fig. 1. 1 .

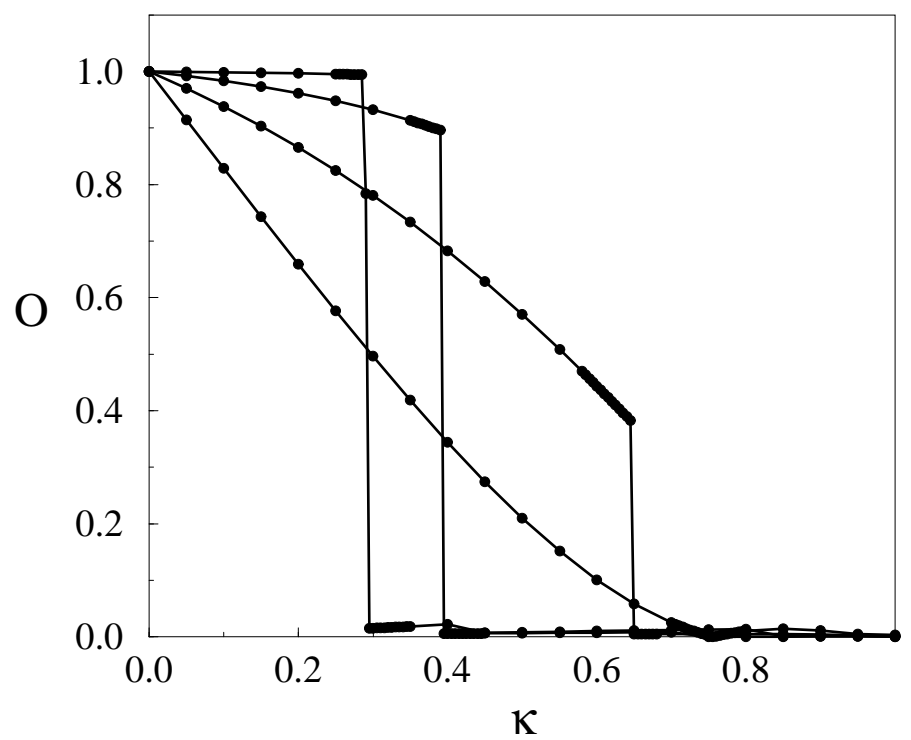

Figure 4: Overlap matrix elements (3.3) for the integrable model with 16 spins as a function of $\kappa$ : the data at small $\kappa$ correspond to states with magnetization $M^{z}=6,4,2,0$ (top to bottom), the lines are guides to the eye. 


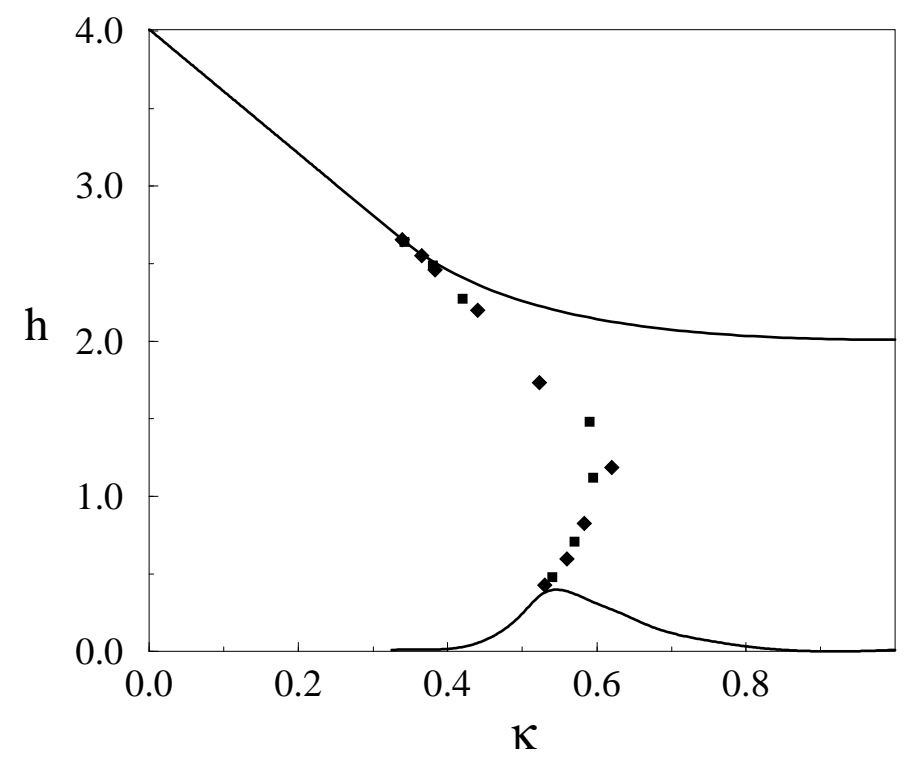

Figure 5: Phase diagram of the spin chain model with nearest and next-nearest neighbour pair exchange $2 J_{2} / J_{1}=\kappa /(1-\kappa)$ : the upper line denotes the saturation field $h_{c 2}$ obtained from spin wave calculation, the lower one denotes the transition at $h=\Delta$ into the dimerized phase with a spin gap $(0.325 \lesssim \kappa<1$ for $h \rightarrow 0)$. Squares (diamonds) denote the transition determined numerically from the overlap criterion (3.3) for systems with 20 (16) spins. 